\title{
Validation and Application of Predictive Models for Inadequate Bowel Preparation in Colonoscopies in a Tertiary Hospital Population
}

\author{
Edgar Afecto Ana Ponte Sónia Fernandes Catarina Gomes \\ João Paulo Correia João Carvalho \\ Department of Gastroenterology, Centro Hospitalar Vila Nova de Gaia/Espinho, Vila Nova de Gaia, Portugal
}

\section{Keywords \\ Colonoscopy · Bowel preparation · Colonoscopy quality criteria}

\begin{abstract}
Background: Bowel preparation is a major quality criterion for colonoscopies. Models developed to identify patients with inadequate preparation have not been validated in external cohorts. We aim to validate these models and determine their applicability. Methods: Colonoscopies between April and November 2019 were retrospectively included. Boston Bowel Preparation Scale $\geq 2$ per segment was considered adequate. Insufficient data, incomplete colonoscopies, and total colectomies were excluded. Two models were tested: model 1 (tricyclic antidepressants, opioids, diabetes, constipation, abdominal surgery, previous inadequate preparation, inpatient status, and American Society of Anesthesiology [ASA] score $\geq 3$ ); model 2 (co-morbidities, tricyclic antidepressants, constipation, and abdominal surgery). $\boldsymbol{R} \boldsymbol{e}$ sults: We included 514 patients (63\% males; age $61.7 \pm 15.6$ years), 441 with adequate preparation. The main indications were inflammatory bowel disease $(26.1 \%)$ and endoscopic treatment (24.9\%). Previous surgery (36.2\%) and ASA score $\geq 3(23.7 \%)$ were the most common comorbidities. An ASA
\end{abstract}

score $\geq 3$ was the only identified predictor for inadequate preparation in this study ( $p<0.001$, OR 3.28). The sensitivity, specificity, positive predictive value (PPV), and negative predictive value (NPV) of model 1 were 60.3, 64.2, 21.8, and $90.7 \%$, respectively. Model 2 had a sensitivity, specificity, PPV, and NPV of $57.5,67.4,22.6$, and $90.5 \%$, respectively. The AUC for the ROC curves was 0.62 for model 1, 0.62 for model 2, and 0.65 for the ASA score. Conclusions: Although both models accurately predict adequate bowel preparation, they are still unreliable in predicting inadequate preparation and, as such, new models, or further optimization of current ones, are needed. Utilizing the ASA score might be an appropriate approximation of the risk for inadequate bowel preparation in tertiary hospital populations.

\footnotetext{
(C) 2021 Sociedade Portuguesa de Gastrenterologia. Published by S. Karger AG, Basel
}

\section{Validação e aplicação de modelos preditivos para preparação intestinal inadequada numa população de um hospital terciário}

Palavras Chave

Colonoscopia · Preparação intestinal · Critérios de qualidade na colonoscopia karger@karger.com www.karger.com/pjg

Karger $\stackrel{\text { ' }}{5}$

BOPEN ACCESS (c) 2021 Sociedade Portuguesa de Gastrenterologia. Published by S. Karger AG, Basel

This is an Open Access article licensed under the Creative Commons Attribution-NonCommercial-4.0 International License (CC BY-NC) (http://www.karger.com/Services/OpenAccessLicense), applicable to the online version of the article only. Usage and distribution for commercial purposes requires written permission.
Correspondence to:

Edgar Afecto, edgarafecto@gmail.com 


\section{Resumo}

Introdução: A preparação intestinal é um dos principais critérios de qualidade na colonoscopia. Modelos desenvolvidos para identificar doentes com preparação inadequada nunca foram validados em coortes externas. Pretendemos validar esses modelos e determinar sua aplicabilidade clínica. Métodos: Colonoscopias entre abril-novembro/2019 foram incluídas retrospectivamente. A Escala de Preparação Intestinal de Boston $\geq 2$ por segmento foi considerada adequada. Dados insuficientes, colonoscopias incompletas e colectomias totais foram excluídos. Dois modelos foram testados: modelo 1 (antidepressivos tricíclicos, opióides, diabetes, obstipação, cirurgia abdominal, preparação prévia inadequada, internamento e American Society of Anesthesiology [ASA] $\geq 3$ ); modelo 2 (comorbilidades, antidepressivos tricíclicos, obstipação e cirurgia abdominal). Resultados: Foram incluídos 514 doentes (63\% homens; idade $61.7 \pm$ 15.6), 441 com preparação adequada. As principais indicações foram doença inflamatória intestinal (26.1\%) e tratamento endoscópico (24.9\%). Cirurgias anteriores (36.2\%) e ASA $\geq 3$ (23.7\%) foram as comorbilidades mais comuns. Um score $A S A \geq 3$ foi o único fator de risco identificado para preparação inadequada ( $p<0.001$, OR 3.28). A sensibilidade, especificidade, valor preditivo positivo (VPP) e valor preditivo negativo (VPN) do modelo 1 foi de $60.3,64.2$, 21.8 e $90.7 \%$. O modelo 2 apresentou sensibilidade, especificidade, VPP e VPN de 57.5, 67.4, 22.6 e $90.55 \%$. A AUC para a curva ROC foi de 0.62 para o modelo 1, 0.62 para o modelo 2 e 0.65 para o score ASA. Conclusões: Embora ambos os modelos sejam eficazes a prever preparação intestinal adequada, não se verifica o mesmo para a preparação inadequada e como tal, novos modelos ou otimização dos atuais são ainda necessários. Utilizar o score ASA pode ser uma aproximação adequada do risco de preparação intestinal inadequada em populações de hospitais terciários.

(c) 2021 Sociedade Portuguesa de Gastrenterologia. Publicado por S. Karger AG, Basel

\section{Introduction}

According to the most recent guidelines on bowel preparation of the European Society of Gastrointestinal Endoscopy (ESGE), the adequacy of bowel preparation is a major quality criterion in colonoscopy [1]. Nevertheless, inadequate bowel preparation is still reported in up to $35 \%$ of patients $[2,3]$.

Predictive Models for Inadequate Bowel Preparation
Two recent systematic reviews and meta-analyses [2, 3] attempted to identify predictive factors for inadequate bowel preparation. Patient age, male gender, medical history (chronic constipation, hypertension, diabetes, cirrhosis, stroke, and dementia), and current medication (opiates and tricyclic antidepressants) were identified as risk factors, while previous abdominal surgery, previous inadequate bowel preparation, and body mass index were not consistent predictors.

Based on these findings, three predictive models [4-6] were developed with the aim of identifying patients at risk for inadequate bowel preparation. To the best of our knowledge, none of these models have yet been applied outside of their development/validation cohorts and in clinical practice.

Therefore, this study aims to validate these inadequate bowel preparation predictive models in our population and to determine their applicability in clinical practice.

\section{Materials and Methods}

Study Population and Data Collection

This study was conducted in a tertiary hospital in northern Portugal. For the purpose of this retrospective cohort study, the authors considered all patients who underwent an elective colonoscopy between April and November 2019. Patient data was collected through database search and clinical records, concerning sex, age, indication for colonoscopy, type of preparation used, American Society of Anesthesiology (ASA) score, simple medical history (diabetes, cirrhosis, neurological disorders, abdominal or pelvic surgery), medication history (opioids, tricyclic antidepressants), previous inadequate bowel preparation, chronic constipation history (defined as fewer than three bowel movements per week), and current hospitalization at the time of colonoscopy.

Bowel preparation was considered adequate if every colon segment scored at least 2 points in the Boston Bowel Preparation Scale (BBPS). The exclusion criteria were absence of BBPS in the exam report, incomplete colonoscopy (for a reason other than inadequate bowel preparation), total colectomy, and incomplete patient data.

\section{Bowel Preparation and Other Interventions}

According to the implemented protocol in our hospital, all patients were provided with written and oral instructions regarding bowel preparation. A low-fiber diet was started in the 2 days prior to the procedure. Per protocol, patients are allowed to choose between low-volume polyethylene glycol (PEG) + ascorbic acid, high-volume PEG or sodium picosulfate (healthy patients, without comorbidities). A split-dose bowel preparation regimen was used for colonoscopies scheduled for the morning period, while a sameday regimen was used for afternoon colonoscopies. Additionally, a nurse was available for a face-to-face consultation with every patient that had doubts or required further instructions regarding bowel preparation regimens. 
Table 1. Patient characteristics and clinical data

\begin{tabular}{|c|c|c|c|}
\hline & & $\begin{array}{l}\text { Univariate, } \\
\text { OR }(95 \% \mathrm{Cl})\end{array}$ & $\begin{array}{l}\text { Multivariate, } \\
\text { OR }(95 \% \mathrm{CI})\end{array}$ \\
\hline \multicolumn{4}{|l|}{ Sex, $n(\%)$} \\
\hline Male & $324(63.0)$ & & \\
\hline Female & $190(37.0)$ & & \\
\hline Age, mean $\pm S D$, years & $61.7 \pm 15.62$ & & \\
\hline \multicolumn{4}{|l|}{ Indications for colonoscopy, $n$ (\%) } \\
\hline IBD & $134(26.1)$ & & \\
\hline Endoscopic treatment & $128(24.9)$ & & \\
\hline Post-polypectomy/cancer follow-up & $125(24.4)$ & & \\
\hline Screening & $41(8)$ & & \\
\hline Iron deficiency anemia & $34(6.6)$ & & \\
\hline Chronic diarrhea & $15(2.9)$ & & \\
\hline Other & $37(7.2)$ & & \\
\hline \multicolumn{4}{|l|}{ Bowel cleanser, $n(\%)$} \\
\hline PEG $2 \mathrm{~L}+$ ascorbic acid & $170(33.1)$ & & \\
\hline PEG 3L & 59 (11.5) & & \\
\hline PEG 4L & $142(27.6)$ & & \\
\hline Sodium picosulfate & $19(3.7)$ & & \\
\hline Unknown & $124(24.1)$ & & \\
\hline Previous abdominal/pelvic surgery, $n(\%)$ & $186(36.2)$ & & \\
\hline Partial colectomy/rectal resection & $77(15)$ & & \\
\hline Urogenital surgery & $60(11.7)$ & & \\
\hline Hepatobiliopancreatic surgery & $15(2.9)$ & & \\
\hline Other & $34(6.6)$ & & \\
\hline ASA score, $n(\%)$ & & $3.50(2.09-5.85)$ & $3.28(2.04-5.28)$ \\
\hline 1 & $32(6.2)$ & & \\
\hline 2 & $358(69.6)$ & & \\
\hline 3 & $123(23.9)$ & & \\
\hline 4 & $1(0.2)$ & & \\
\hline \multicolumn{4}{|l|}{ Co-morbidities, $n$ (\%) } \\
\hline Diabetes & $110(21.4)$ & $2.35(1.38-4.02)$ & \\
\hline Cirrhosis & $6(1.2)$ & & \\
\hline Chronic constipation & $71(13.8)$ & $2.17(1.18-4.02)$ & \\
\hline Stroke and/or dementia & $24(4.7)$ & & \\
\hline \multicolumn{4}{|l|}{ Chronic medication used, $n$ (\%) } \\
\hline Opioids & $9(1.8)$ & $5.05(1.32-19.23)$ & \\
\hline Tricyclic antidepressants & $7(1.4)$ & & \\
\hline Inpatient colonoscopy, n (\%) & $22(4.3)$ & & \\
\hline Previous inadequate preparation, $n(\%)$ & $55(10.7)$ & $2.06(1.04-4.07)$ & \\
\hline
\end{tabular}

Only significant values are presented. $\mathrm{OR}$, odds ratio; $\mathrm{Cl}$, confidence interval; SD, standard deviation; IBD, inflammatory bowel disease; PEG, polyethylene glycol; ASA, American Society of Anesthesiology.

Bowel Preparation Predictor Models

Two predictive models, previously published, were tested in our population. Model 1 by Dik et al. [5] considers tricyclic antidepressants or opioids use, diabetes, constipation, previous abdominal surgery, previous inadequate preparation, inpatient status, and ASA score $\geq 3$. Model 2 by Gimeno-García et al. [6] considers comorbidities (diabetes, cirrhosis, and stroke history), tricyclic antidepressants use, constipation, and previous abdominal surgery. A third model, developed by Hassan et al. [4], was not tested as it did not use a validated bowel preparation scale.
Statistical Analysis

Continuous variables are expressed as medians and interquartile ranges (IQR), while categorical variables are expressed as frequencies and percentages. All potentially inadequate bowel preparation factors were subjected to univariate ( $\chi^{2}$ for categorical and $t$ test for continuous variables) and multivariate analysis (logistic regression). Two-tailed $p$ values were considered statistically significant if $<0.05$. A subset analysis comparing high- and low-volume PEG was performed regarding ASA score, age, comorbidities, constipation, previous surgery, current inpatient status, and previous colonoscopy with inadequate preparation as we postulated 
Table 2. Bowel preparation

\begin{tabular}{ll}
\hline Bowel preparation, $n(\%)$ & \\
$\quad$ Adequate & $441(85.8)$ \\
$\quad$ Inadequate & $73(14.2)$ \\
Total BBPS, mean \pm SD & $7.04 \pm 1.85$ \\
$0-2, n(\%)$ & $10(1.9)$ \\
$3-5$ and 6 (at least 1 segment <2), $n(\%)$ & $63(12.3)$ \\
6-9, $n(\%)$ & $441(85.8)$ \\
BBPS per segment, mean \pm SD & \\
Right colon & $2.28 \pm 0.72$ \\
Transverse colon & $2.44 \pm 0.67$ \\
Left colon & $2.32 \pm 0.67$ \\
\hline
\end{tabular}

that old patients with more comorbidities were probably being offered more high-volume PEG preparations due to safety concerns.

The discriminative power of both models in predicting inadequate bowel preparation was determined by calculating the sensitivity, specificity, positive predictive value (PPV), negative predictive value (NPV), and the area under the curve (AUC) of the receiver operating characteristic (ROC) curves.

The $\chi^{2}$ test of goodness-of-fit was performed to evaluate whether the sample size was adequate, using the G*Power software and data available in the literature $[5,6]$. This analysis revealed that a power of $95 \%$ for model 1 would have been achieved with 169 patients, while for model 2, enrolling 423 patients would allow for a power of $90 \%$; in the present study, 514 individuals were included, allowing adequate power to test both models.

All statistical analyses were performed in IBM SPSS Statistics v22.0 (SPSS Inc., Chicago, IL, USA) and G*power v3.1.9.7 (Heinrich-Heine-Universität Düsseldorf, Germany).

\section{Results}

We included 514 patients, 324 (63.0\%) of which were males. The median age was 64 years (IQR 53-73.25). The most common indications for colonoscopy were inflammatory bowel disease $(n=134,26.1 \%)$, endoscopic treatment $(n=128,24.9 \%)$, and cancer/polypectomy followup $(n=125,24.4 \%)$. Previous abdominal/pelvic surgery was the most common comorbidity $(n=186,36.2 \%)$, followed by diabetes $(n=110,21.4 \%)$. The majority $(n=371$, $72.2 \%$ ) of patients underwent bowel cleansing with PEGbased solution. A more extensive description of patient clinical data and baseline characteristics can be found in Table 1.

Adequate bowel preparation, as defined in the Methods section, was observed in $441(85.8 \%)$ patients. The median total BBPS score was 7 points (IQR 6-9), and the median right colon, transverse colon, and left colon segment scores were 2,3 , and 2 points, respectively. This data is summarized in Table 2.

Predictive Models for Inadequate Bowel Preparation

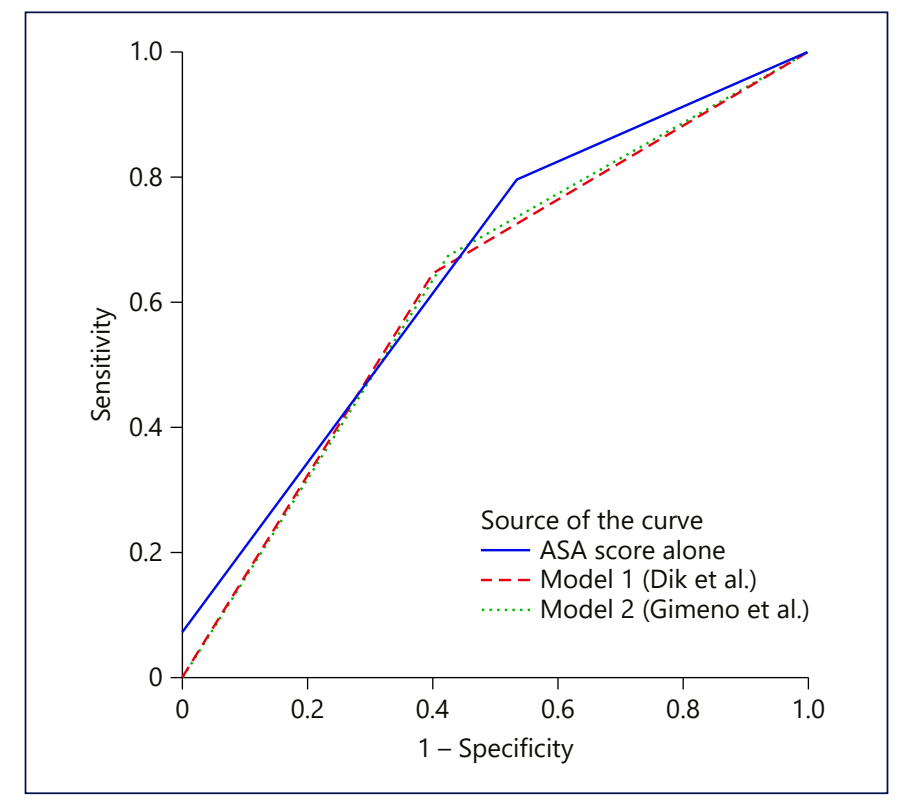

Fig. 1. ROC curves and AUC of both models and the ASA score.

On bivariate analysis, we found an association between inadequate preparation and opioid use $(p=0.027)$, diabetes mellitus $(p=0.001)$, chronic constipation $(p=$ $0.011)$, previous inadequate preparation $(p=0.034)$, and ASA score $(p<0.001)$. On further multivariate analysis, the same effect was observed only for those with higher ASA scores $(p<0.001$, OR 3.28, 95\% CI 2.04-5.28). No association was found regarding age, sex, volume of PEG used (high versus low volume), tricyclic antidepressant use, cirrhosis, neurologic comorbidities, previous surgery (even when separated by colon resection vs. other intraabdominal surgeries), and inadequate bowel preparation. This information is also displayed in Table 1.

Model 1 predicted a total of 202 patients as having inadequate bowel preparation, 44 of which were correctly predicted as such. On the other hand, 312 were predicted as adequate preparation, 283 of which were correctly predicted. The sensitivity, specificity, PPV, and NPV of model 1 were $60.3,64.2,21.8$, and $90.7 \%$, respectively. The AUC for the ROC curve of this model was 0.62 (95\% CI $0.55-0.69$ ).

Model 2 predicted 186 patients as having inadequate bowel preparation, 42 of which were correctly predicted as such. As for adequate preparation, 328 were predicted to achieve it, but only 297 did so. Model 2 had a sensitivity, specificity, PPV, and NPV of 57.5, 67.4, 22.6, and $90.6 \%$, respectively. The AUC for the ROC curve of this 
model was 0.62 (95\% CI 0.55-0.70). ROC curves for both models are shown in Figure 1.

A subset analysis comparing high- and low-volume PEG regimens showed that higher ASA scores $(p=0.001)$ and inpatient status $(p=0.008)$ were significantly associated (on bivariate and multivariate analysis) with the use of higher-volume PEG regimens. Higher age was also associated with higher-volume PEG on bivariate analysis, but this was not confirmed on multivariate analysis.

Lastly, as the ASA score was the only predictive factor in our study, we tested its accuracy in predicting inadequate preparation. Utilizing an ASA score $>2,124$ patients were predicted as having inadequate preparation, 34 of which were correctly predicted. On the other hand, 390 were predicted as having adequate preparation, 351 of which were correctly predicted. This translates as a sensitivity, specificity, PPV, and NPV of 46.6, 79.6, 27.4, and $90.0 \%$, respectively. The AUC for the ASA score was 0.65 ( $p<0.001,95 \%$ CI $0.58-0.72$ ). This ROC curve is also presented in Figure 1.

\section{Discussion}

In this study, we were able to replicate the data published by the authors of these scores, with similar, although slightly worse, results $[5,6]$.

The ongoing search for factors that can influence bowel preparation and accurately identify these patients resulted in these two previously published scores. Although the results seemed promising, some limitations are easily identified and were further confirmed in our study. More than half of the patients with inadequate preparation were identified, but there is still a significant group of patients that were not identified by either score $(39.7 \%$ for model 1 and $42.5 \%$ for model 2) and in which we thus could not intervene. Although the argument can be made that all patients identified by these scores as having inadequate preparation could be offered more intensive regimens, it is also true that we would be subjecting a nonnegligible number of patients to unnecessary interventions (which could further reduce compliance), as is demonstrated by the observed very low PPVs and high false positives for both scores. As such, the scores demonstrated a low value in predicting inadequate bowel preparation.

On the other hand, both scores were found to have a very high NPV, which means they could be useful in determining which patients most likely do not require additional interventions.
When comparing the original model 1 study, by Dik et al. [5], some methodological similarities and differences can be pointed out. While most of our exams were performed in an inflammatory bowel disease or endoscopic treatment setting, the original study included mostly patients undergoing screening or symptom investigation, which may induce a difference in adhesion to the bowel cleansing protocols due to different populations. Additionally, in this study, BBPS was defined as inadequate if total $<6$, and no reference was made to segments scoring 1 point with a total of 6 , which we considered as inadequate preparation in our paper. Although the majority of patients in both our study and the original study are ASA class 1 or 2 , while Dik et al. [5] only had $4.5 \%$ of patients with ASA $\geq 3$, we had $24.1 \%$ scoring $>2$ points, which can be explained by an overall increased prevalence of comorbidities in our population. Overall, while there are differences in the populations being compared, model 1 performed worse in our study, with lower sensitivity ( $0.60 \mathrm{vs.}$ $0.66)$, specificity (0.79 vs. 0.64$), \operatorname{PPV}(0.22$ vs. 0.29$)$, and NPV (0.91 vs. 0.95$)$.

Regarding the original study for model 2, by GimenoGarcía et al. [6], similar limitations can be described. Most examinations were also performed in a screening/ symptom investigation setting, with equivalent conclusions regarding applicability. In this study, the median age of patients (60 vs. 64 in our study) was lower and comorbidities were present in a lower proportion (21.8 vs. $24.1 \%)$. Patients with dementia and previous history of inadequate bowel preparation were excluded in this study but included in our study because we believe these patients are at greater risk of inadequate bowel preparation. Additionally, the proportion of opioid ( 4.8 vs. $1.8 \%$ ) or tricyclic antidepressant (8.2 vs. $1.4 \%$ ) use was significantly higher in their population. On the other hand, as was the case with the model 1 study [5], the study by GimenoGarcía et al. [6] was prospective in nature, contrasting with our retrospective study and its inherent biases. With these differences summarized, differences in model 2 accuracy were expected and were observed as a higher sensitivity ( 0.58 vs. 0.50$)$ and NPV (0.91 vs. 0.88$)$ but a lower specificity ( 0.67 vs. 0.80$)$ and PPV (0.23 vs. 0.36$)$.

Conversely, chronic constipation and abdominal surgery were not identified as predictors. This could be explained by the retrospective nature of our study, mostly in the case of chronic constipation, as we could not always use objective definitions for these categories due to incomplete data. In concordance with both studies $[5,6]$, we considered both partial colectomies and other intraabdominal/pelvic surgeries the same for the purpose of 
this study, and, as such, different relationships between surgeries and inadequate preparation can arise (as we do not know what surgeries the patients in the original studies had in order to make a comparison). Additionally, a sub-analysis regarding type of surgery (colon/rectum resection or urogenital surgery) found no relationship with inadequate preparation. Nevertheless, the relationship between previous surgery and inadequate preparation is controversial, as two meta-analyses with a significant number of patients failed to demonstrate any relationship $[2,3]$, probably due to the heterogeneity of definitions used and patients included.

The models previously described are probably more useful outside of tertiary specialized hospitals, as the population in our study (older, more comorbidities, and lower proportion of screening/symptom investigation) demonstrated substantially different results and lower AUC values than previously published.

In terms of previously identified inadequate bowel preparation predictors, we were only able to identify higher ASA scores as a predictive factor for an inadequate preparation in our population. By utilizing the ASA score alone, we demonstrated a predictive power similar but slightly better to the two models tested (AUC 0.65 vs. 0.62 on both models tested). The ASA score is widely used and easy to apply in clinical practice and categorizes patients according to their comorbidities. In populations such as ours (a tertiary hospital), utilizing the ASA score in order to triage patients who should be paid more attention regarding bowel preparation is an easier and quicker method than the two scores analyzed in this paper.

When aiming to optimize bowel preparation, several steps should be taken by all patients. Patients should adopt a clear-liquid or low-fiber diet on the previous day as both are equally effective $[7,8]$, although a low-fiber diet is associated with higher tolerability and willingness to repeat the exam. Bowel preparation should be undertaken as a split-dose regimen for next-day procedures or a same-day regimen for afternoon procedures $[1,9,10]$. The bowel preparation utilized (high-volume PEG, lowvolume PEG, or non-PEG regimen if clinically validated) can be chosen according to patient preference, as there seems to be no difference in efficacy between regimens [11]. High-volume regimens offer better safety profiles with the trade-off of diminished tolerability (and thus more inadequate preparations), which might be more relevant in older patients with more comorbidities [12].

With all these previous measures applied, bowel preparation is more likely to be optimized, even in patients who are thought to be at risk (such as ASA >2). Neverthe-

Predictive Models for Inadequate Bowel Preparation less, additional measures, such as enhanced bowel preparation instructions, should be applied - such as a face-toface or telephone nursing consultation $[13,14]$. Although it may seem reasonable to prescribe additional laxatives or high-volume preparations in constipated individuals, the current available evidence shows no difference between regimens in these patients [15]. In case of a previous inadequate preparation, a modifiable reason for the failure of the chosen regimen should be sought before prescribing a different regimen or additional measures, such as nausea/vomiting or poor adherence due to patient- or preparation-related factors.

Several limitations can be readily identified, mostly due to the retrospective nature of our study and its inherent biases, such as the likelihood of suffering from missing data. Patient compliance or tolerance to bowel preparation was not registered, but nevertheless, our reported proportion of adequate bowel preparation was similar to previously published literature $[5,6]$ and nearly achieved the $\geq 90 \%$ recommended threshold [16], which probably indicates an adequate (but not perfect) compliance. Additionally, not all preparation regimens were registered, as this was not practice in out hospital at this time period, but it is reasonable to assume that most of these patients underwent a PEG solution, although the proportion of which cannot be inferred due to missing data. Regarding volume of preparation, the utilization of higher-volume PEG solutions was higher in older patients (although not significantly so), inpatients, and higher ASA scores: all inpatients are prescribed higher-volume PEG, as it is the only solution available in our hospital; as for higher ASA and age, we postulate that due to comorbidities and advanced age, these patients were probably recommended by the nursing staff or the community pharmacist to undergo higher-volume preparations for safety reasons. Although this may introduce a bias, we believe it not to be significant since the proportion of adequate preparation was the same between groups (high vs. low volume). Lastly, in our population, 26.1 and $24.9 \%$ of colonoscopies were performed in inflammatory bowel disease and endoscopic therapy settings, which limits the generalization of our data and indicates that these scores are not suitable for a tertiary hospital population such as ours.

In conclusion, although both models are capable of predicting more than half of the patients with inadequate bowel preparation, they are unable to do so in a reliable manner and, therefore, almost half of those requiring more intensive regimens are not identified when using these models. Further improvement of these models or development of new ones is necessary before they can be 
applied in clinical practice. Utilizing the ASA score might be an appropriate approximation of the risk for inadequate bowel preparation in tertiary hospital populations.

\section{Statement of Ethics}

The study protocol was approved by the local ethics committee. The research was conducted ethically in accordance with the Declaration of Helsinki 2014 [9].

\section{Conflict of Interest Statement}

The authors have no conflicts of interest to declare.

\section{Funding Sources}

This work was not funded.

\section{Author Contributions}

Edgar Afecto: Inception, design and drafting of the article. Ana Ponte: Inception, design and critical review of the article. Sónia Fernandes: critical review of the article and final approval. Catarina Gomes: data collection and critical review of the article. João Correia: data collection and critical review of the article. João Carvalho: critical review of the article and final approval.

\section{References}

1 Hassan C, East J, Radaelli F, Spada C, Benamouzig R, Bisschops R, et al. Bowel preparation for colonoscopy: European Society of Gastrointestinal Endoscopy (ESGE) Guideline - Update 2019. Endoscopy. 2019 Aug;51(8):77594.

2 Gandhi K, Tofani C, Sokach C, Patel D, Kastenberg D, Daskalakis C. Patient characteristics associated with quality of colonoscopy preparation: a systematic review and metaanalysis. Clin Gastroenterol Hepatol. 2018 Mar;16(3):357-369.e10.

3 Mahmood S, Farooqui SM, Madhoun MF. Predictors of inadequate bowel preparation for colonoscopy: a systematic review and meta-analysis. Eur J Gastroenterol Hepatol. 2018 Aug;30(8):819-26.

4 Hassan C, Fuccio L, Bruno M, Pagano N, Spada C, Carrara S, et al. A predictive model identifies patients most likely to have inadequate bowel preparation for colonoscopy. Clin Gastroenterol Hepatol. 2012 May;10(5):501-6.

5 Dik VK, Moons LM, Hüyük M, van der Schaar P, de Vos Tot Nederveen Cappel WH, Ter Borg PC, et al.; Colonoscopy Quality Initiative. Predicting inadequate bowel preparation for colonoscopy in participants receiving split-dose bowel preparation: development and validation of a prediction score. Gastrointest Endosc. 2015 Mar;81(3):665-72.
6 Gimeno-García AZ, Baute JL, Hernandez G, Morales D, Gonzalez-Pérez CD, NicolásPérez D, et al. Risk factors for inadequate bowel preparation: a validated predictive score. Endoscopy. 2017 Jun;49(6):536-43.

7 Nguyen DL, Jamal MM, Nguyen ET, Puli SR, Bechtold ML. Low-residue versus clear liquid diet before colonoscopy: a meta-analysis of randomized, controlled trials. Gastrointest Endosc. 2016 Mar;83(3):499-507.e1.

8 Avalos DJ, Sussman DA, Lara LF, Sarkis FS, Castro FJ. Effect of Diet Liberalization on Bowel Preparation. South Med J. 2017 Jun;110(6):399-407.

9 Martel M, Barkun AN, Menard C, Restellini S, Kherad O, Vanasse A. Split-Dose Preparations Are Superior to Day-Before Bowel Cleansing Regimens: A Meta-analysis. Gastroenterology. $2015 \mathrm{Jul} ; 149(1): 79-88$.

10 Cheng YL, Huang KW, Liao WC, Luo JC, Lan $\mathrm{KH}$, Su CW, et al. Same-day Versus Split-dose Bowel Preparation Before Colonoscopy: A Meta-analysis. J Clin Gastroenterol. 2018 May/Jun;52(5):392-400.

11 Xie Q, Chen L, Zhao F, Zhou X, Huang P, Zhang L, et al. A meta-analysis of randomized controlled trials of low-volume polyethylene glycol plus ascorbic acid versus standard-volume polyethylene glycol solution as bowel preparations for colonoscopy. PLoS One. 2014 Jun;9(6):e99092.
12 Ho SB, Hovsepians R, Gupta S. Optimal Bowel Cleansing for Colonoscopy in the Elderly Patient. Drugs Aging. 2017 Mar;34(3):16372.

13 Gaspar R, Andrade P, Ramalho R, Antunes J, Macedo G. Bowel preparation: modifiable factors to improve bowel cleansing. Eur J Gastroenterol Hepatol. 2019 Jan;31(1):140.

14 Guo X, Yang Z, Zhao L, Leung F, Luo H, Kang $X$, et al. Enhanced instructions improve the quality of bowel preparation for colonoscopy: a meta-analysis of randomized controlled trials. Gastrointest Endosc. 2017 Jan;85(1):9097.e6.

15 Parente F, Vailati C, Bargiggia S, Manes G, Fontana P, Masci E, et al. 2-Litre polyethylene glycol-citrate-simethicone plus bisacodyl versus 4-litre polyethylene glycol as preparation for colonoscopy in chronic constipation. Dig Liver Dis. 2015 Oct;47(10):857-63.

16 Kaminski MF, Thomas-Gibson S, Bugajski M, Bretthauer M, Rees CJ, Dekker E, et al. Performance measures for lower gastrointestinal endoscopy: a European Society of Gastrointestinal Endoscopy (ESGE) Quality Improvement Initiative. Endoscopy. 2017 Apr;49(4):378-97. 